\title{
SUMMARY OF PHOTON 2001
}

\author{
ARMIN BÖHRER \\ Fachbereich Physik, Universität Siegen, 57068 Siegen, Germany \\ E-mail: armin.boehrer@cern.ch \\ MARIA KRAWCZYK \\ IFT UW, Hoza 69, 00-681 Warszawa, Poland \\ E-mail: Maria.Krawczyk@fuw.edu.pl
}

\begin{abstract}
This article summarizes the experimental and theoretical results presented and discussed at the International Conference on the Structure and Interactions of the Photon, PHOTON 2001, in Ascona.
\end{abstract}

\section{Introduction}

The photon, the first and best known gauge boson, is a fundamental particle with pointlike couplings to charged fundamental particles. In high energy hadronic processes, however, the photon may behave like a hadron. The study of the structure and the interaction of the photon in soft, semi-hard and hard hadronic processes turns out to be a rich field of investigation and test of the strong interaction. Detailed studies are performed especially at $\mathrm{e}^{+} \mathrm{e}^{-}$colliders and now also with ep interactions. Different in their initial state, processes at LEP and HERA add to give a common understanding of the photon and its interaction. This is rounded off by specific, detailed and complementary results at low energy colliders also discussed at this conference.

In this summary we present the highlights of the conference PHOTON 2001 as seen by the two authors. For further details we refer the reader to the individual presentations or the group summaries of the conference and references therein.

\section{Theoretical Framework}

The soft high-energy processes involving photons, a bulk of events, can be described in the VDM-type models using a framework of Regge Theory, while in the hard processes the perturbative QCD works; the semi-hard processes corresponds to the case where both Regge approach and pQCD are applicable. In both, hard and semi-hard processes the notion of the partonic structure of the photon is useful and it is clear that in such processes the photon may participate directly or via its partonic agents (the resolved photon processes).

summary-ascona-final: submitted to World Scientific on March 9, 2022 1 
The standard DGLAP approach to describe hard processes involving photons, like DIS on the photon and large $p_{T}$ jet (particle) production in $\gamma \gamma$ or $\gamma \mathrm{p}$ collision, is based on the collinear approximation where terms with large $\log Q^{2}$ or $\log p_{T}^{2}$ are summed up. The leading and next-to-leading (LO and NLO) calculations exists for many quantities, also all existing parton parametrizations for the photon were obtained in such an approach.

For semi-hard processes, e.g., DIS at small $x$, there appear other large scale and corresponding large logarithms have to be summed to all orders. Resummation of large $\log 1 / x$ terms can be obtained using BFKL or CCFM evolution equations. In both of the latter approaches the $p_{t}$ of the initial parton cascade is not ordered; the basic ingredients are the unintegrated parton densities to be convoluted with appropriate matrix elements with the initial parton being off-shell. These approaches were presented during this conference, being superior in some cases to the standard DGLAP approach in the description of the data.

In many respects the approaches used to describe photons are similar to ones used for a hadron, e.g., proton. One should be aware, however, that the possible direct coupling to a pointlike parton influences significantly the whole picture. The Parton Model prediction can be derived for $F_{2}^{\gamma}$ from the basic process $\gamma^{*} \gamma \rightarrow \mathrm{q} \overline{\mathrm{q}}$ giving a logarithmic dependence on $Q^{2}$. The corresponding DGLAP evolution equations, which take into account additional QCD radiation processes are inhomogeneous, and in principle (unfortunately not in practice due to appearance of singularities) can be solved without any input (asymptotic solution). Basic ideas and results can be found in recent reviews 2 . 2.3 .

\section{Structure Functions}

The (hadronic) structure function $F_{2}^{\gamma}$ of the (real) photon is measured in the hadron production processes in unpolarized $\mathrm{e}^{+} \mathrm{e}^{-}$collisions from the single-tagged events. The negative momentum transfer squared of the photon emitted by the tagged lepton is equal to $q^{2}=-2 E_{\text {tag }} E_{\text {beam }}(1-\cos \theta)$. The second lepton stays undetected so the corresponding momentum transfer squared $p^{2}=-P^{2}$ is small. The process then can be viewed as a deep inelastic electron-photon scattering $\left(\mathrm{DIS}_{\mathrm{e} \gamma}\right)$, where a quasi-real photon (with "mass squared" $p^{2}$ ) is probed by a virtual photon with high virtuality $Q^{2}=-q^{2}$. The differential cross section can be expressed as $\mathrm{d}^{2} \sigma_{\mathrm{e} \gamma \rightarrow \mathrm{eX}} / \mathrm{d} x \mathrm{~d} Q^{2}=\left[\left(1+(1-y)^{2}\right) F_{2}^{\gamma}\left(x, Q^{2}\right)-y^{2} F_{L}^{\gamma}\left(x, Q^{2}\right)\right] 2 \pi \alpha^{2} / x Q^{2}$ with $x \approx Q^{2} /\left(Q^{2}+W^{2}\right)$ and the inelasticity $y \approx 1-\left(E_{\mathrm{tag}} / E_{\mathrm{beam}}\right) \cos ^{2} \theta_{\mathrm{tag}}$.

In most cases $y$ is small and the term with $F_{L}^{\gamma}$ can be neglected. Some

summary-ascona-final: submitted to World Scientific on March 9, 2022 2 
experiments correct their results for it, with the increased precision of the measurement the correction should become mandatory, as it was noted at the conference. The e $\gamma$ cross section given above is used to extract the structure function $F_{2}^{\gamma}\left(x, Q^{2}\right)$ primarily related to the quark densities in the photon $q^{\gamma}\left(x, Q^{2}\right)$, see Ref. 4 and Ref. 3 .

The measurements of $F_{2}^{\gamma}$ at LEP reached $x$ as low as $10^{-3}$ and cover a range of $\left\langle Q^{2}\right\rangle$ from about $2 \mathrm{GeV}^{2}$ to more than $750 \mathrm{GeV}^{2}$ (even above 1000 $\mathrm{GeV}^{2}$ for $Q^{2}$-dependence). All existing data are shown in Fig. 1 as a function of $x$ the new data obtained at the highest $Q^{2}$ by OPAL and DELPHI groups 5 are shown in the last diagram. The logarithmic rise with $Q^{2}$ seen in data is predicted by the theory. It appears already in the Parton Model QCD modifies this rise softly (logarithmically). In Fig. 3 the evolution with $Q^{2}$ is shown for various $x$ regions (DELPHI) in comparison with other data, MC predictions and with few parametrizations. The data (Fig. 11) are nicely described by the GRV-HO and SaS-1D parton parameterizations for a real photon; with the present precision the $Q^{2}$ dependence data (Fig. 33) start to challenge existing parton parametrizations and MC models. Note, that all parametrizations for photon were obtained from data collected before 1997. The full present set of over two hundred data points is being used in a new LO fit to $F_{2}^{\gamma}$ based on DGLAP evolution equation 7 . The updated parton parametrization for a real photon can be used to describe hard processes induced by the real photons at LEP and HERA, where some discrepancies are being observed (see Sec. 3 and 4). This new parametrization will be important also for studies of potential of future linear colliders, especially the photon colliders (see Sec. 7).

An extraction of $F_{2}^{\gamma}$ by fitting its components (QPM, VDM and RPCresolved photon contribution) separately, rather than a regularized unfolding, may lead to a better measurement; the estimated uncertainties increase but are more reliable, as stated by DELPHI 6 . It has been pointed out at the conference, however, that improvements are still to be made: where precision is high by including the $P^{2}$ dependence or statistics is low by combining all four LEP experiments. Note that if $P^{2}$ differs from zero, we deal with a virtual photon, which has the three states of polarization: two transverse(like a real photon) and one longitudinal (called also scalar) ones, see Sec. 3.1 where the importance of the resolved $\gamma_{L}^{*}$ contribution are discussed.

The charm contribution to the photon structure function $F_{2}^{\gamma}$, denoted $F_{2, \mathrm{c}}^{\gamma}$, can be obtained from the $\mathrm{DIS}_{\mathrm{e} \gamma}$ data from the sample with charmed hadrons. The updated result of OPAL, which identifies charm by exclusive reconstruction of a $\mathrm{D}^{*}$ meson (see also below), strengthens their previous finding that for $x<0.1$ the predicted hadron-like contribution is lower than

summary-ascona-final: submitted to World Scientific on March 9, 2022 3 

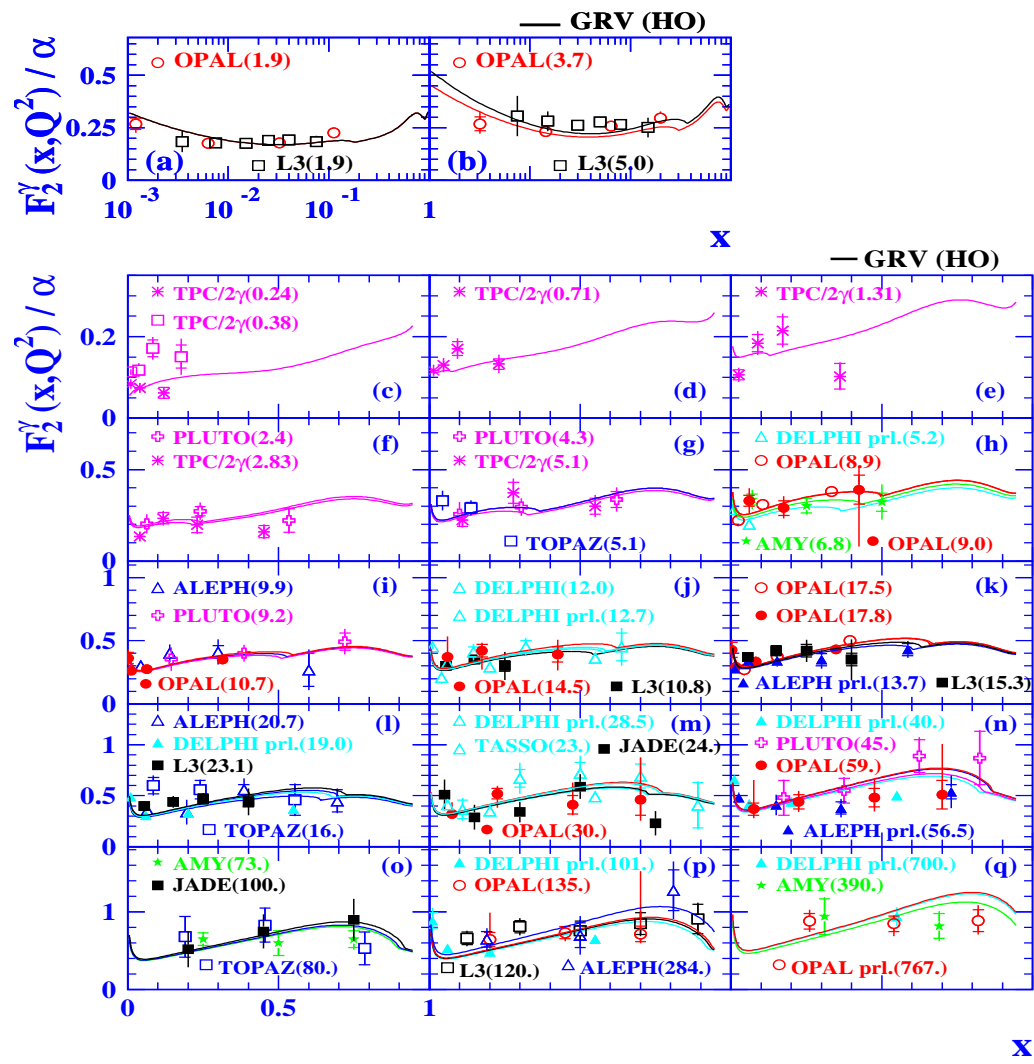

Figure 1. Summary of measurements of the hadronic structure function $F_{2}^{\gamma}$, from $⿴$.

the measurement $\mathrm{O}$, see Fig. 3. A confirmation of the difference by the other LEP experiments is desirable. More data are needed to constrain the gluonic content of the photon in such measurements.

A first measurement has also been performed by OPAL of the process $\mathrm{e} \gamma \rightarrow \mathrm{eZ} / \gamma^{*}$ where the centre-of-mass energy of the $e Z / \gamma^{*}$-system $\sqrt{\hat{s}}$ is equal or larger than the $\mathrm{Z}$ mass $\mathrm{G}$ and found to be in agreement with $\mathrm{MC}$ predictions.

\section{Hard Inclusive Processes}

Hard production of jets (single jet and dijets) or particles at LEP and HERA are used as a complementary method to probe the "structure" of the pho- 


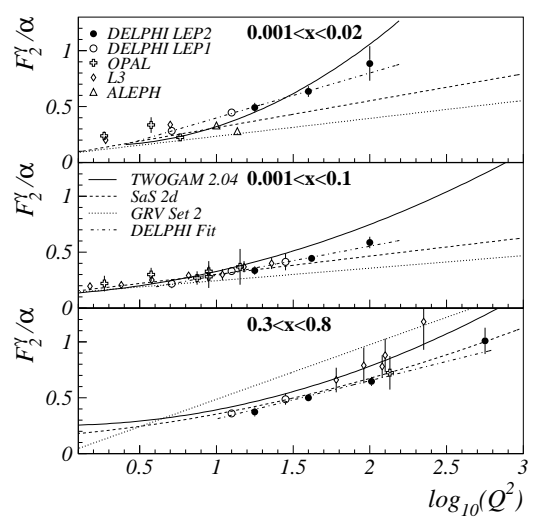

Figure 2. Fvolution of $F_{2}^{\gamma}$ with $Q^{2}$ in various $x$ - regions 6 .
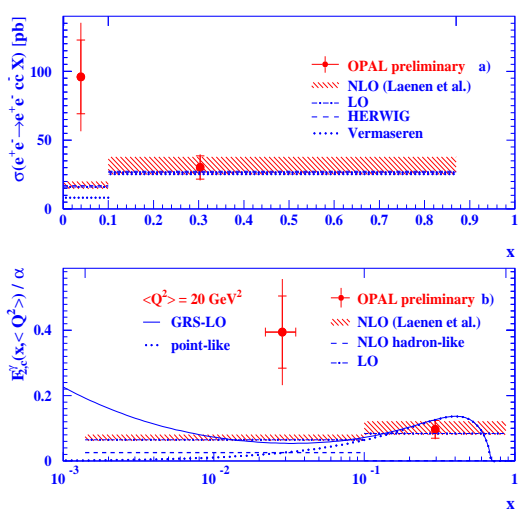

Figure 3. Results for $F_{2, c}^{\gamma}$ 目.

ton 10 . The hard scale $\tilde{Q}$ is then provided by a large $p_{T}$ of produced jets or particles. The part of momentum carried by a partonic constituent of the photon, $x_{\gamma}$, is at LO equal to $x$. Direct, single-resolved (for LEP also doubleresolved) photon processes contribute, and can be separated at LO by using the fact that in the resolved one the remnant jet carries away a part of the invariant mass available in the $\gamma \gamma$ or $\gamma p$ collision. For dijets at HERA the variable $x_{\gamma}$, can be reconstructed from the pseudorapidity $\eta$ and transverse momentum $E_{T}$ of the jets, as $x_{\gamma}=\left(E_{T}^{\mathrm{jet} 1} \mathrm{e}^{-\eta^{\mathrm{jet} 1}}+E_{T}^{\mathrm{jet} 2} \mathrm{e}^{-\eta^{\mathrm{jet} 2}}\right) /\left(2 y E_{\mathrm{e}}\right)$. Similarly $x_{\gamma}^{ \pm}$relevant for LEP measurements (photon-photon collisions) can be obtained.

The implementation of NLO in the event simulation for jet production is in progress as was reported at this conference, where a systematic method for combining NLO QCD calculation with the parton was presented 12. Special emphasis was recently put on the study of the (soft) underlying events (sue), especially important for the double-resolved contribution. Inclusion of multiple parton interaction (MIA), parametrized as in pure hh collision, usually improves the agreement with data.

The structure of jets, forming subjets, has been investigated at HERA 11 , where samples of different gluon purity have been obtained in events with charm quarks. The subjet structure is predicted by QCD as a function of the jet resolution parameter. The results are consistent with perturbative QCD and consistent with findings in hadronic $\mathrm{Z}$ decays at LEP. Further tests of 
QCD such as the measurementof $\alpha_{\mathrm{S}}$ give values compatible in precision with other measurements or better 13.

Both at LEP (see Ref. 14 for reviews) and HERA inclusive hadron production has been studied intensively. Predictions from MLLA, e.g., average charged multiplicity, Gaussian shape of the distribution in $\xi=$ $-\ln \left(p_{\text {hadron }} / p_{\text {beam }}\right)$ and the dependence of the maximum $\xi^{*}$ on $Q^{2}$, were shown at this conference for HERA and found in very good agreement with the data both in deep inelastic and diffractive scattering processes, respectively, proving also the universality of the fragmentation functions. Such studies were performed for charged particles inclusively, for strange particles and particles versus anti-particles 15 (see also Ref. 16 for similar studies in charmed jets at HERA).

\subsection{Jets}

- Dijets at LEP

Present measurements of dijet events at LEP, from a $\sim 600 \mathrm{pb}^{-1}$ data sample collected, concentrate on the separation of the direct and (single)resolved contribution to investigate the gluon content of the real photon 17 . $\mathrm{t}$, which is not tightly constrained by the $F_{2}^{\gamma}$ data.

Comparisons with the NLO calculations and the Monte Carlo models PYTHIA and HERWIG are made by OPAL for $E_{T}$, and $\eta$ as well as $x_{\gamma}^{ \pm}$distributions (see Fig. 4). The MC predictions for both parton parametrizations, GRS or SaS-1D, are too low by $20 \%$ for small $x_{\gamma}$ and small $E_{T}$ indicating a too low gluon content in the photon. To study the effects of the underlying event the data sample is divided into samples where $x_{\gamma}^{+}$or $x_{\gamma}^{-}<0.75$ and $>0.75$, giving data sets with different single- and double-resolved fraction.

Similar studies were also performed by DELPHI 6 for $E_{T}$ and jet profile distributions. Jet profiles turn out to be very sensitive to the presence of possible MIA effects. PYTHIA with MIA (default setting) and HERWIG with MIA (sue $=20 \%$, i.e., in $20 \%$ of the simulated double-resolved events a soft underlying event was included) are favoured.

The OPAL and DELPHI data, for which comprehensive studies and Monte Carlo comparisons have been presented, are consistent with each other. Note however that cqmparisons to the NLO QCD calculation were made on parton level only 17 and it had been pointed out previously that hadronization corrections are important. The differential cross section in $E_{t}^{\mathrm{jet}}$ and $\eta$ need corrections of $10 \%$ to $20 \%$. However, for the distributions in $x_{\gamma}$, very sensitive to the hadronisation, the corrections may be even higher.

summary-ascona-final: submitted to World Scientific on March 9, 2022 6 

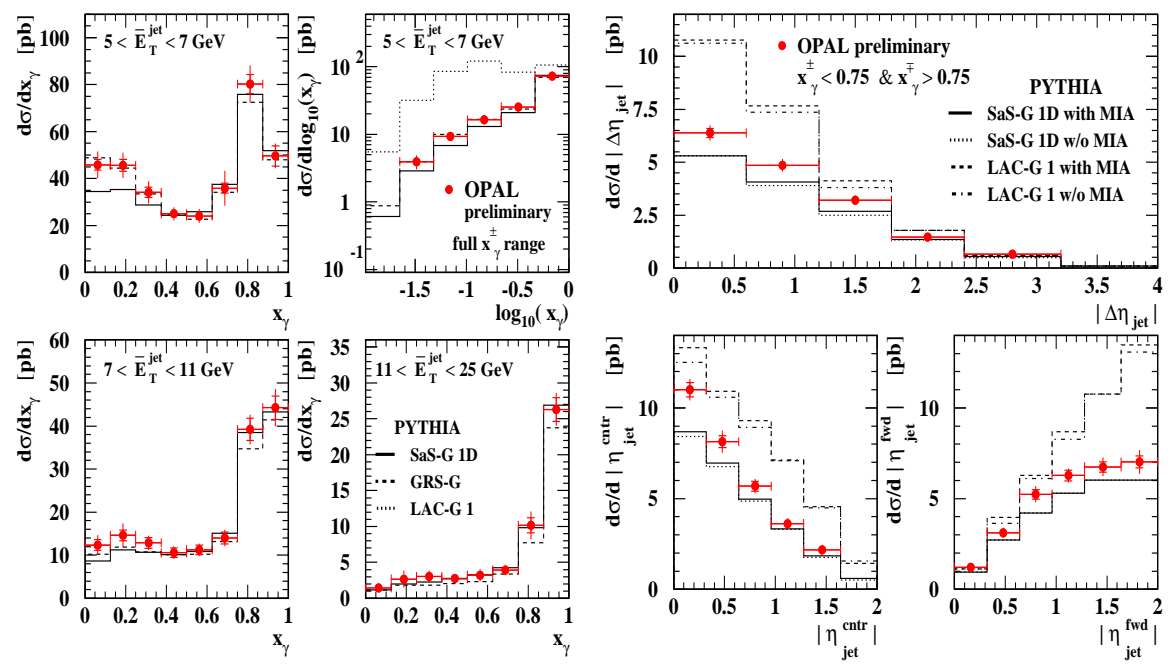

Figure 4. The dijet cross section (OPAL) as a function of $x_{\gamma}$ for the various mean transverse energies of the dijet system and as a function of $\Delta \eta 17$.

-Jet and dijets production at HERA in photoproduction and DIS $\mathrm{ep}_{\mathrm{ep}}$ events

At HERA using the jet data, parton densities of the proton and of the photon can be constrained. The structure of the real photon (photoproduction, $Q^{2}<1 \mathrm{GeV}^{2}$ ) and of the virtual photon (electroproduction or DIS $\mathrm{ep}$ events with $Q^{2}>1 \mathrm{GeV}^{2}$ ) have been studied, including direct and resolved photon contributions. In the resolved virtual photon processes at HERA the variable $Q^{2}$ is the virtuality (mass squared) of the photon, which structure is tested by large $p_{T}$ jets or particles, $p_{T}^{2} \gg Q^{2}$. For the first time a partonic content of $\gamma_{L}^{*}$ was included in the analysis of the HERA data, see below. On the other hand interference terms, which may be important, as it was addressed in 19, were omitted in the analysis.

H1 and ZEUS have measured cross section for the photoproduction of inclusive jet as well as dijet as a function of $\eta$ and $E_{T}$ 20. The ZEUS and H1 data are in nice agreement with each other in most observables (after correction for different cuts) and with the calculations for inclusive jet production, see Fig. F-left and center, where H1 data are shown. However for dijets event ZEUS finds a deficit at low $x_{\gamma}$ (even for large $E_{T}$ sample) indicating that more 

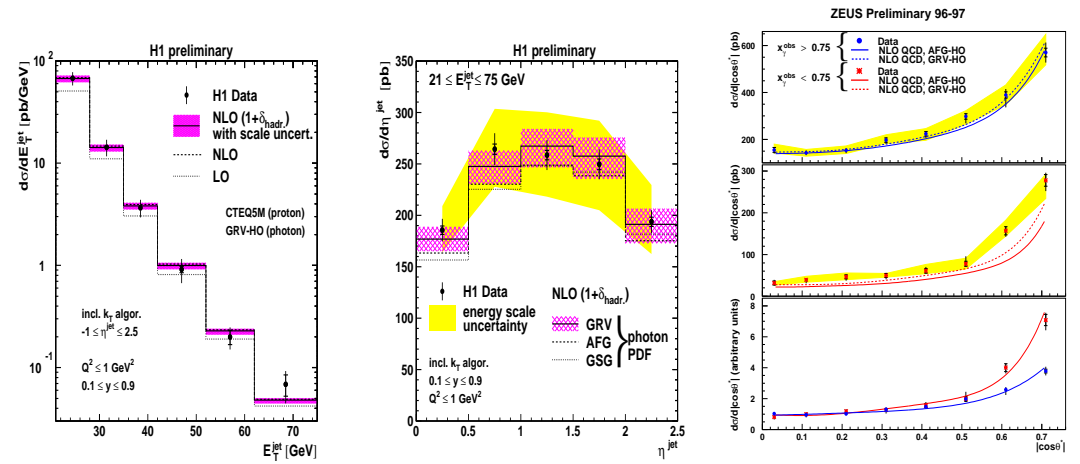

Figure 5. Left and Centre: The jet cross sections $\mathrm{H} 1$ data in comparison with the NLO calculations for different parton parametrizations 20 ;

Right: The dijet cross section (ZEUS) as a function of $\left|\cos \theta^{*}\right|$, from 20 .

gluons are needed in the photon. It is demonstrated in Fig. p-right, where the angular distribution is presented for various subprocesses. The $\mathrm{H} 1$ dijet data differ here from the ZEUS measurements and do not show a discrepancy with the NLO predictions. It was pointed out that the NLO calculations for cuts chosen for dijet events by ZEUS (e.g., different $E_{T}^{\min }$ for the two jets) should be more reliable 10 .

The partonic content of the virtual photon has been investigated at HERA in dijet events, confirming the main $\ln p_{T}^{2} / Q^{2}$ dependence of the parton densities for $\gamma^{*}$ predicted by the theory (already by Parton Model). This behaviour leads to a suppression of the cross section with growing $Q^{2}$ for a fixed $p_{T}$ value (range). ZEUS results, in form of a ratio of the cross sections for events with $x>0.75$ and $x<0.75$, are presented in Fig. 6-left (below similar ratio for subsample with charm will be shown). The ratio, plotted as a function of $Q^{2}$ for various $E_{T}$ ranges, shows disagreement with HERWIG predictions (with SAS1D, GRV). H1 data in two similar samples were compared with HERWIG MC predictions based on the direct $\gamma^{*}$ and on the resolved $\gamma_{T}^{*}$ contribution. When in addition the contribution due to the partonic content of the longitudinally polarized virtual photon is included in analysis 21 , the description of the data is improved. Here also the CASCADE Monte Carlo based on CCFM evolution was applied - it gives prediction even closer to the data (Fig. 6-right), however the $Q^{2}$ dependence is not well reproduced 21.

summary-ascona-final: submitted to World Scientific on March 9, 2022 8 

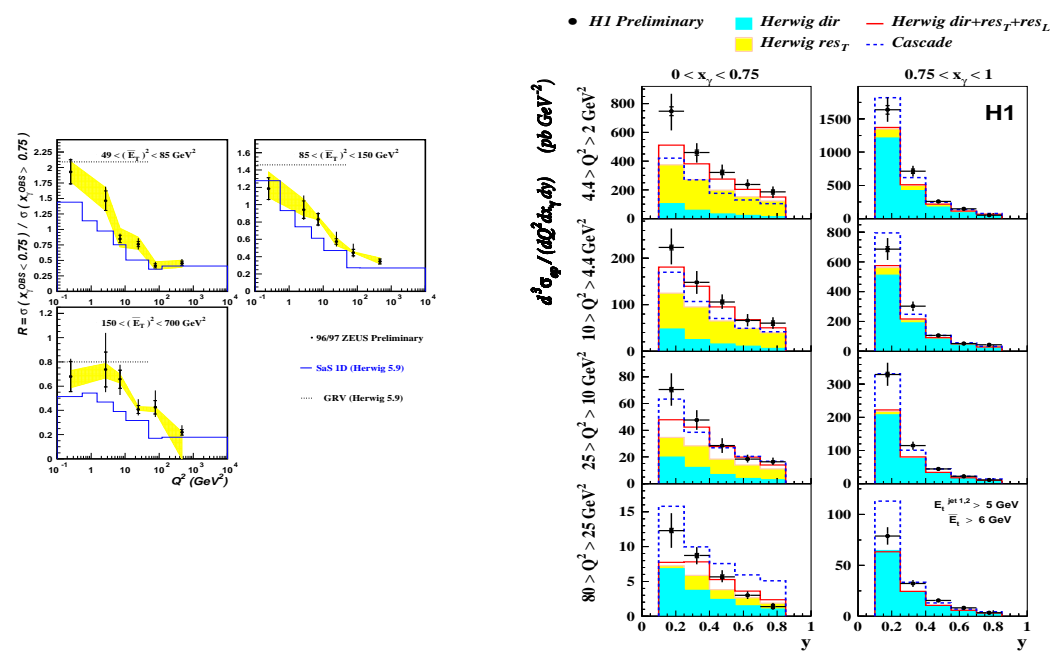

Figure 6. Left: The ratio of cross sections $R=\sigma\left(x_{\gamma}<0.75\right) / \sigma\left(x_{\gamma}>0.75\right)$, from ZEUS, as a function of $Q^{2} 21$;

Right: Dijet cross section for the $\mathrm{H} 1 \mathrm{data}$, the dark (light) histogram represents the direct $\gamma^{*}$ (direct plus the resolved $\gamma_{T}^{*}$ ) contribution of HERWIG. The full line stands for the sum of the direct $\gamma^{*}$ and of $\gamma_{T}^{*}$ and $\gamma_{L}^{*}$ resolved photon contributions of HERWIG; the dashed line is CASCADE prediction 21 .

\subsection{Prompt Photons}

At the Tevatron, HERA and recently also at LEP collider the prompt (isolated) photon production has been studied 22 23. While for $\mathrm{p} \overline{\mathrm{p}}$ collisions the D0 data agree with the NLO predictions, CDF observes an excess at low photon $p_{T}$, similar as in measurements at lower centre-of-mass energy in hadronhadron collisions, see Fig. 7-left. The $k_{t}$ smearing for initial partons helps to describe data. The run II at Tevatron may clarify the situation, see also 24 where plans of future measurements of prompt photons at LHC are discussed. Note, that such processes are sensitive to the gluonic content of the proton. At the ep-collider HERA the photoproduction of the prompt photon plus jet were measured by the ZEUS group recently (in previous publications ZEUS 25 dealt with prompt photons only; then, their data showed slight disagreement with the NLO calculation in the $\eta$ distribution for small $y$ sample 26). In this new measurement a direct photon sample, ie. with $x_{\gamma} \sim 1$, was used to

summary-ascona-final: submitted to World Scientific on March 9, 2022 9 

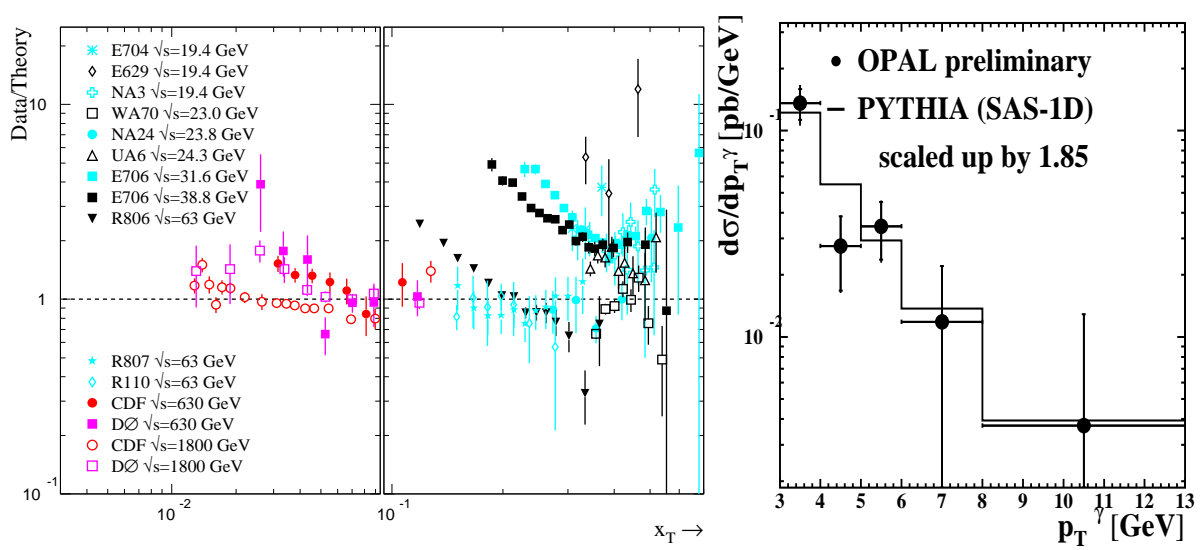

Figure 7. The prompt photon production. Left: At hadron-hagtron colliders in comparison with NLO QCD prediction as function of $x_{T}=2 E_{T} / \sqrt{s}$, from 22;

Right: At LEP as a function of $p_{T}$ in comparison with PYTHIA results 23.

extract the $\left\langle k_{t}\right\rangle, \sim 2 \mathrm{GeV}$, in the proton 27 . The new OPAL measurements of the inclusive production of the isolated photon in the process $\gamma \gamma \rightarrow \gamma \mathrm{X}$ needs a $K$-factor of 1.85 to describe the data (Fig. 1 -right) 23.

\subsection{Hadron Production}

In Fig. $8 p_{T}$ spectra for $\pi^{0}$ and $\mathrm{K}_{\mathrm{S}}$ in two-photon collisions are presented as measured by L3. The data show the expected exponential behaviour for small $p_{T}$, for $p_{T}>1.5 \mathrm{GeV}$ a power law $p_{T}^{-B}$ with $B \approx 4$ indicates a simple underlying 2 body $\rightarrow 2$ body process. The spectra are also compatible with NLO calculation or PHOJET prediction (which are similar) for momenta up to a few $\mathrm{GeV}$. Beyond $5 \mathrm{GeV}$, where data only for $\pi^{0}$ are available a clear disagreement is seen: the NLO prediction lies below the data points (Fig. 8 - 2 , PHOJET exceeds the measurements while PYTHIA is below (not shown) 28 . It was pointed out that further tests for charged pions are welcome.

\section{Charm and Beauty Production}

Heavy quark production provides important tests of QCD. It gives complementary to jets information on partonic densities in the photon. Although the large quark mass should allow for reliable perturbative calculations in

summary-ascona-final: submitted to World Scientific on March 9, 2022 10 

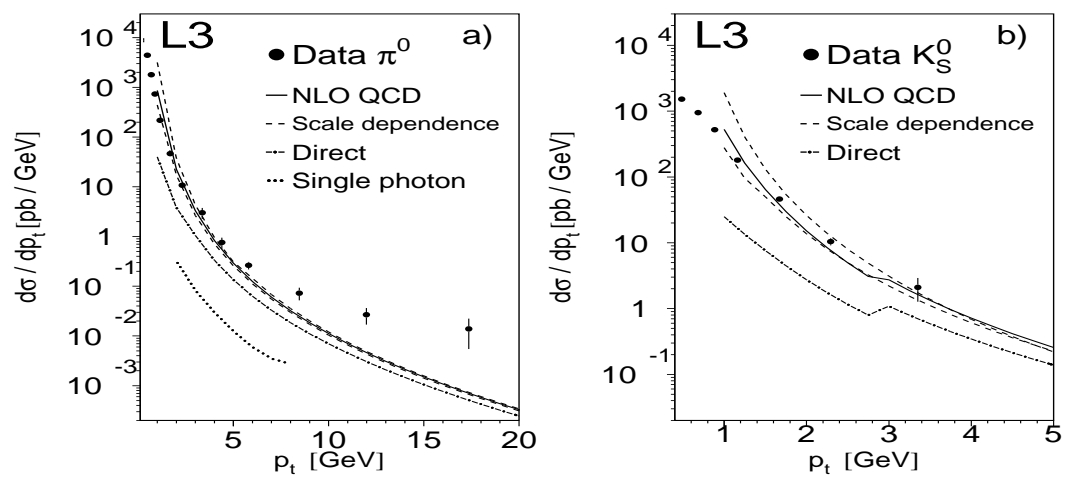

Figure 8. Inclusive differentiatcross section $\mathrm{d} \sigma / \mathrm{d} p_{T}$ for (a) $\pi^{0}$ and (b) $\mathrm{K}_{\mathrm{S}}$ in comparison with NLO QCD calculations 28.

QCD, discrepancies are observed for the heavy quark productions, especially for $\mathrm{b}$ production, at all colliders. The question may appear on a limitation of the standard calculation based on a DGLAP evolution, both for massless and massive approaches used in the calculations. It has been pointed out at this conference that a recently developed CASCADE program based on the CCFM evolution equations using unintegrated parton distributions reproduces the $\mathrm{b}$ production cross section at the Tevatron 29 . CASCADE also reproduces most charm and bottom distributions at HERA, unfortunately it has not been applied for $\gamma \gamma$ processes.

For beauty production the QCD predictions are expected to be more reliable than for charm, but are still limited by the smaller cross section. More considerations for a better description of in the $\mathrm{b}$ sector vere debated (e.g., possible need of additional terms 30 ). It was emphasized 31 that care should be taken, whether comparisons are performed for B meson spectra or b-jet spectra, with the latter being more closely related to the partons.

A clear, non-ambiguous signal for charm quark production is the presence of a $\mathrm{D}^{*+}$. This gold-plated signature has been exploited by all four LEP and both HERA collaborations. The leptonic decay has been used by ALEPH ( $\mu^{ \pm}$for charm), L3 ( $\mu^{ \pm}$and $\mathrm{e}^{ \pm}$both for charm and bottom) and OPAL ( $\mu^{ \pm}$for bottom). Though these analyses rely on the momentum spectrum as theoretical input the statistics is substantially increased as compared to the $\mathrm{D}^{*+}$ analyses 32.33 . The $\mathrm{b}$ measurements at HERA rely on the inclusive semileptonic decays, using electrons or muons in dijet events. This signature is combined either with the requirement of a large $p_{\mathrm{T}}^{\text {rel }}$ to a jet (exploiting the

summary-ascona-final: submitted to World Scientific on March 9, 2022 11 

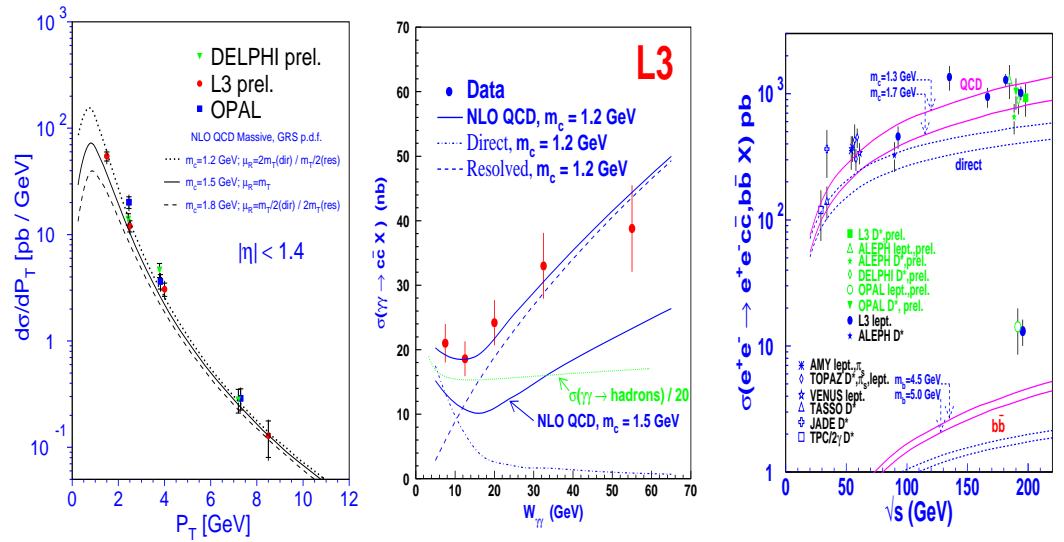

Figure 9. Left: Differential cross section for $\mathrm{D}^{*}$ production obtained by LEP experiments in comparison with NLO QCD calculations, Centre: Charm production in $\gamma \gamma$ collision; Right: Summary of open chatrn and bottom production at $\mathrm{e}^{+} \mathrm{e}^{-}$colliders in comparison with NLO QCD calculations 32 .

effect of the large b quark mass), or the secondary vertices (profiting from the lifetime of the B mesons).

\subsection{Heavy Flavour Production at LEP}

As discussed above for jets the production of heavy flavour in two-photon collisions is dominated by two processes, the direct and the single-resolved process. Both contribute in equal shares to heavy flavour final states at LEP 2 energies. The LEP experiments, ALEPH, DELPHI, L3, and OPAL, agree among themselves and with the theoretical expectation, which e.g., predicts a flat distribution in pseudorapidity for $\mathrm{D}^{*}$ production. In transverse momentum distribution the measurements are close to the NLO QCD prediction based on a massive quarks approach, while there is some scatter among data, Fig. 9 right. The experiments measure the fraction of direct and single-resolved contribution on a $10 \%$ level and confirm the prediction of NLO calculations 31 .

The charm cross section as function of $W_{\gamma \gamma}$ (Fig. 9-centre) shows a steeper rise as compared to $\sigma(\gamma \gamma \rightarrow$ hadrons $)$, see Sec. 5 . With $m_{\text {charm }}=1.2 \mathrm{GeV}$ the agreement with NLO QCD calculation is very good and also shgws that the contribution from the resolved process dominates at high $W_{\gamma \gamma} 43$.

The present status of the measurements for the total $\mathrm{e}^{+} \mathrm{e}^{-} \rightarrow \mathrm{e}^{+} \mathrm{e}^{-} \mathrm{c} \overline{\mathrm{c}}, \mathrm{b} \overline{\mathrm{b}} \mathrm{X}$

summary-ascona-final: submitted to World Scientific on March 9, 2022 12 

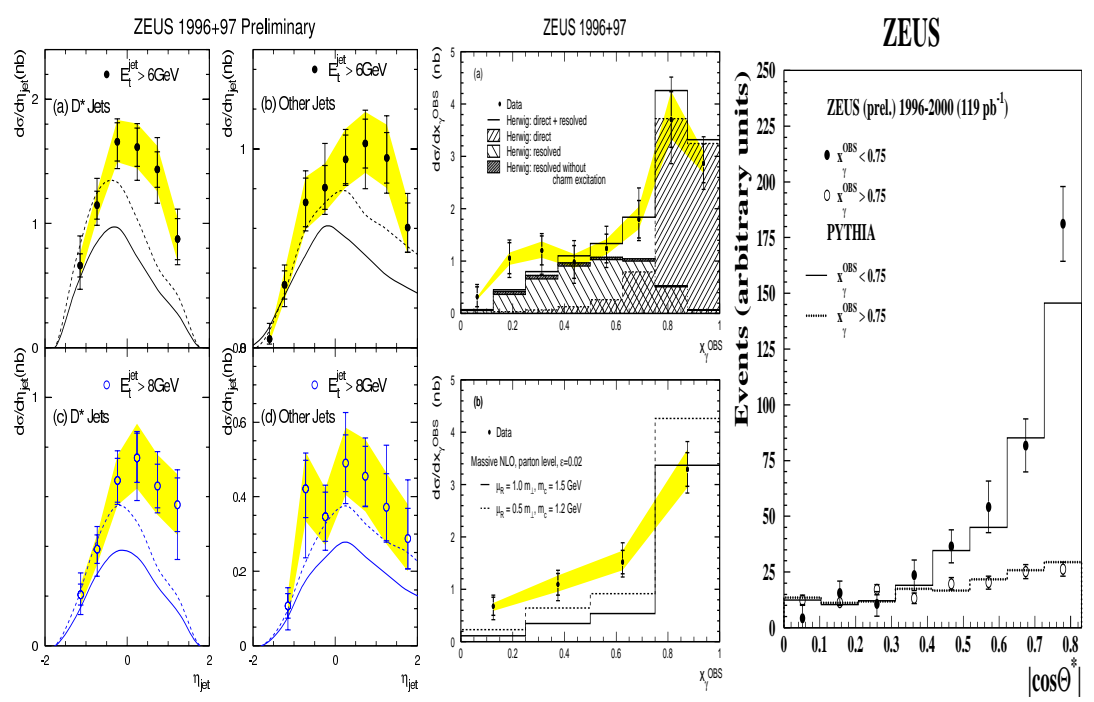

Figure 10. Charm in photoproduction (ZEUS). Left: Data for charm and non-charm jets; Centre: Results for dijets for with a reconstructed $\mathrm{D}^{*}$ meson in comparison with the HERWIG model for $x_{\gamma}$ distribution (a) and with a parton level NLO QCD calculation (b); Right: The $\cos \theta^{*}$ distributions for dijets events vith additional cuts; direct and resolved $\gamma$ samples in comparison with the PYTHIA model 16 .

cross section is summarized in Fig. 9-right. The importance of the resolved photon contribution is obvious for both charm and bottom production. The results are compared to NLO calculations, and good agreement is seen for charm production while a large discrepancy $(3-4 \sigma)$ is observed for bottom. Bottom production is measured by L3 using the fact, that the momentum as well as the transverse momentum of leptons with respect to the closest jet is higher for muons and electrons from bottom than for background, which is mainly charm. The total bottom production cross section is extracted correcting for detector efficiency. Note a large sensitivity of the cross section to the value of heavy quark mass. The same findings on agreement with theory, but with the studies restricted to the muonic channel, come from OPAL. This experiment also investigated the $x_{T}=2 p_{T} / W_{\text {vis }}$ distribution, where $p_{T}$ is the transverse momentum of the muon with respect to the beam axis; the shape of the MC simulation is found compatible with the data 32.

summary-ascona-final: submitted to World Scientific on March 9, 2022 13 

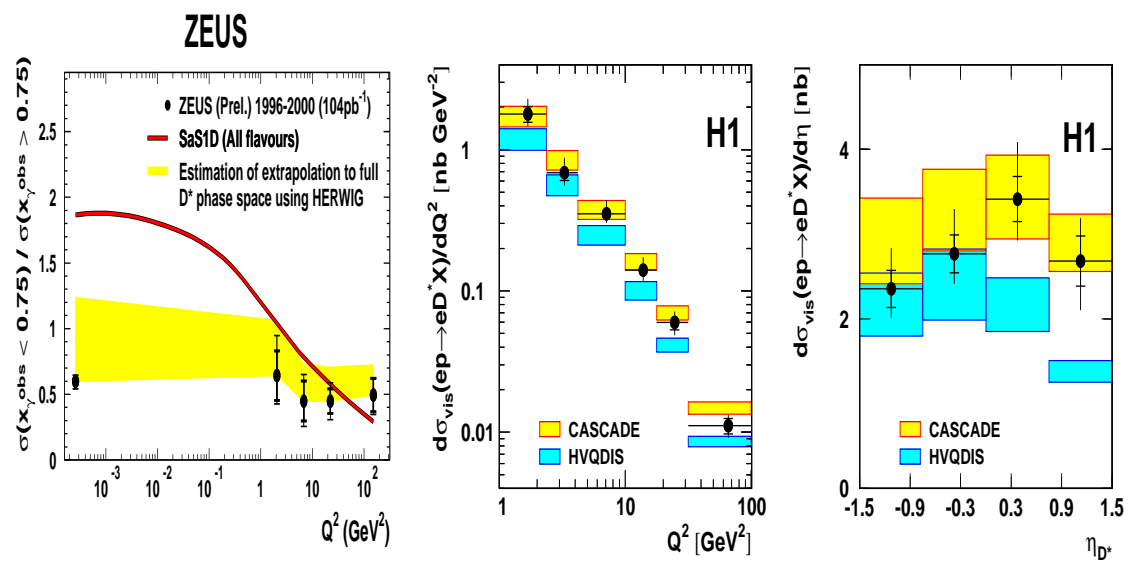

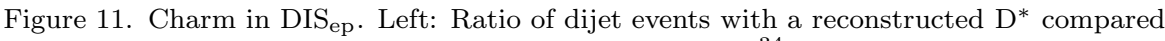
to predictions of the SaS-1D parton parametrization for $\gamma$ 34; Centre: Results for $\mathrm{D}^{*}$ productimn as a function of $Q^{2}$;

Right: as in center for $\eta$, from 35 .

\subsection{Heavy Flavour Production at HERA}

The charm production (jets and $\mathrm{D}^{*}$ mesons) are measured by $\mathrm{H} 1$ and ZEUS both for a real and virtual photon interaction with a proton. Although such processes are dominated by the photon-gluon fusion, which directly probes the gluon content of the proton (or the pomeron for the diffractive events) they also offer opportunity to study the resolved photon contributions.

The cross section as measured by ZEUS in photoproduction as a function of pseudorapidity for charm and non-charm jets are shown in Fig. 10-left. The failure of the NLO QCD to describe the data in the forward hemisphere is evident. The difference of shape and normalization can not be explained by the fragmentation, not included in the simulation 16 . A similar effect is seen in Fig. 10-centre; HERWIG and NLO QCD fail to describe the data for low $x_{\gamma}$, where the resolved photon contribution dominates. Nevertheless, checking the underlying dynamics by the $\cos \theta^{*}$ distribution, with additional cuts (e.g., $M_{J J}>18 \mathrm{GeV}$ ), separately for direct (large $x_{\gamma}$ ) and resolved (low $x_{\gamma}$; gluon exchange) enriched samples, good agreement with the PYTHIA simulation is found, see Fig. 10-right.

For the virtual photon the ratio of low to high $x_{\gamma}$ in dijet events as function of $Q^{2}$ (Fig. 11-left) was measured by ZEUS, and suppression with

summary-ascona-final: submitted to World Scientific on March 9, 2022 14 

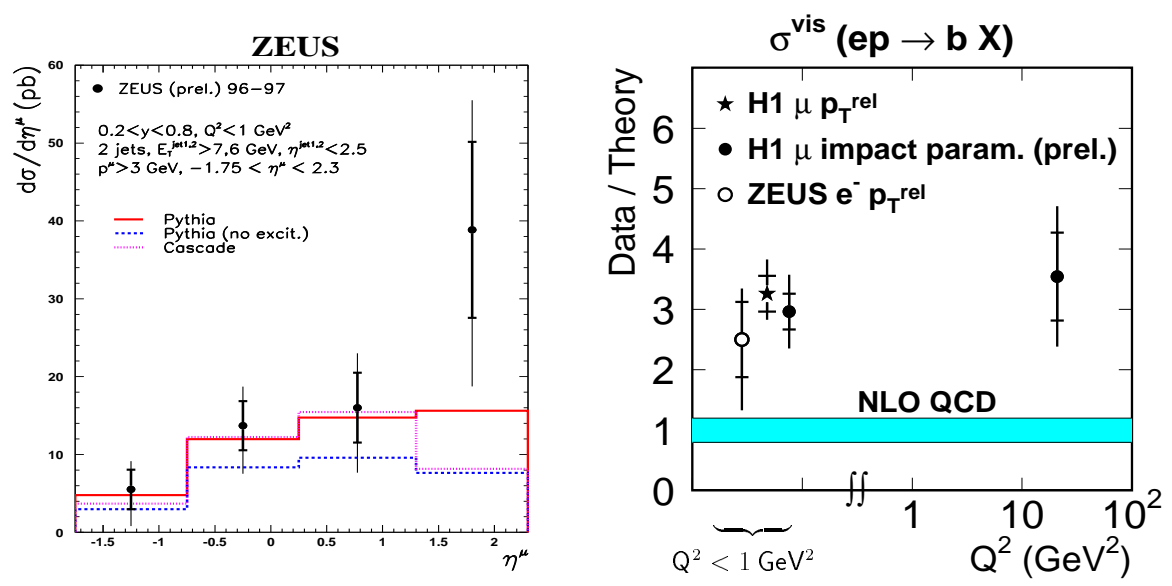

Figure 12. Left: ZEUS data for mapidity distribution for $\mathrm{b}$ in photoproduction in comparison to PYTHIA and CASCADE 29;

Right: Comparison of megsured b production cross section at HERA to NLO QCD calculation as function of $Q^{2} 36$.

$Q^{2}$ was found, weaker however than for the all-flavours case 34. Suppression with growing $Q^{2}$ is observed also in $\mathrm{H} 1$ data for $\mathrm{D}^{*}$ production, see Fig. 11 . centre.

In $\mathrm{DIS}_{\mathrm{ep}}$ events the measurements of $\mathrm{H} 1$ and ZEUS for $\mathrm{D}^{*}$ production for the total cross section and the differential distributions in $p_{T}^{\mathrm{D} *}$ and $\eta^{\mathrm{D} *}$ are nicely described by CASCADE. The HVQDIS Monte-Carlo, traditionally used in ep-scattering for heavy flavour production, fails in describing the forward $\left(\eta_{\mathrm{D} *}\right)$ range giving too low cross section (Fig. 11-right), similarly problem is seen in photoproduction events (Fig.10-left).

The total cross section for $\mathrm{b}$ production has been studied both in photoproduction, and in DIS $_{\text {ep }}$ events. ZEUS results for photoproduction are presented in Fig. 12 -left for a distributions of the $\mu$ coming from b-quark decays. There is an agreement with CASCADE prediction, except in the forward direction. Here the $k_{t}$-factorization may break down 29. Note, that in this region $x_{\mathrm{p}}$ is here rather large, while $x_{\gamma}$ is small.

The visible cross section and the total cross section from Monte Carlo simulations have been used to convert the b quark cross section from restricted $p_{\mathrm{T}}$ and $\eta$ ranges to form the $Q^{2}$ and $y$ distributions. The standard (DGLAP) NLO QCD calculations give results being lower than the measure-

summary-ascona-final: submitted to World Scientific on March 9, 2022 15 

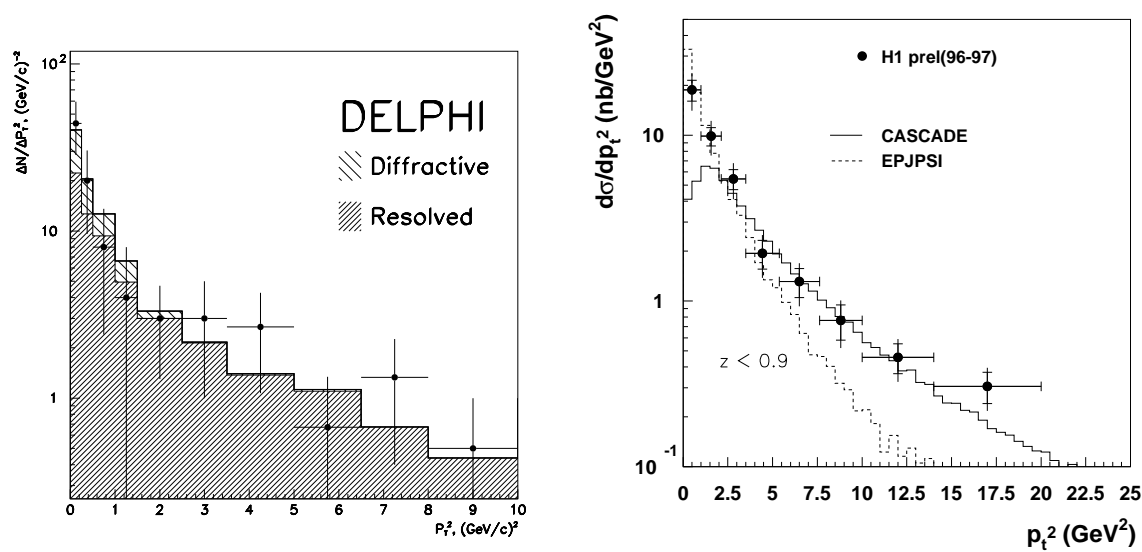

Figure 13. Left: DELPHI data for differential cross section $\mathrm{d} \sigma / \mathrm{d} p_{T}^{2}(\mathrm{~J} / \psi)$ 33; Right: $\mathrm{H} 1$ data for $\mathrm{d} \sigma / \mathrm{d} p_{T}^{2}(\mathrm{~J} / \psi)$ in compared to MC 29.

ments as shown in Fig. 12-right. CASCADE is above these calculations, but still somewhat below the data 36 .

\subsection{Inclusive $J / \psi$ Production}

In two-photon collisions at LEP a first look to $\mu^{+} \mu^{-}+\mathrm{X}$ has been given where the muons are the decay products of the $\mathrm{J} / \psi$, as shown in Fig. 13 33-left. DELPHI finds $36 \pm 7$ events in the muonic decay. The $p_{T}$ spectrum allows for the separation of the resolved (gluon exchange) and diffractive process (pomeron exchange). The latter fraction is found to $26 \pm 22 \%$. A precise measurement may help understand the total $\gamma \gamma$ cross section. Recently 07 , it was proposed that a comparison with theoretical calculations of $\mathrm{J} / \psi$ plus two jets may show, if colour octet production is important as it was found at Tevatron and at LEP in $\mathrm{e}^{+} \mathrm{e}^{-}$annihilation events.

In continuum events taken by BaBar (SLAC) close to the $\Upsilon(4 S)$ both cross section and polar angular distribution of inclusiee $\mathrm{J} / \psi$ production favour NRQCD fragmentation with the colour octet model 38 .

The production of $\mathrm{J} / \psi$ at HERA (H1) is also shown in Fig. 13 -right in agreement with the CASCADE model.

summary-ascona-final: submitted to World Scientific on March 9, 2022 16 


\section{Total Cross Sections for $\gamma \gamma$ and $\gamma^{*} \gamma^{*}$. Diffraction}

Pure perturbative QCD can not describe soft and semi-hard inclusive processes at very high energy. The old, very well known and successful framework for soft phenomena is based on the Regge models, with exchange of Pomeron and Reggeons. The Pomeron is a complicated object with vacuum quantum numbers; its exchange governs the elastic and diffractive processes at high energies or at small $x$. Other processes and quantities, such as the difference of the total cross sections for $\mathrm{pp}$ and $\mathrm{p} \overline{\mathrm{p}}$ collisions, is thought to be related to the exchange of the Odderon, a partner of the Pomeron but with opposite $C$ and $P$ quantum numbers. There are various attempts to apply the perturbative QCD to the small $x$ regions. Simple models based on the two-gluon and three-gluon exchange to represent Pomeron and Odderon exchange.

Important progress has been obtained recently in this field, new theoretical and experimental results were presented $39.40,41,42$. For a consistent description at small $x$ in the BFKL approach, the impact factors of $\gamma^{*}$ at NLO are needed 39. The advantage of the impact factors is that they can be calculated from first principles in the perturbative QCD (the photon-Reggeon interaction).

The only place at present to test Odderon exchange processes is the HERA collider. A dedicated study of photoproduction with exclusive production of mesons decaying into photonic final states - with even or odd number of photons (from two to five photons) - has been performed by H1 and no sign of an Odderon contribution was found 42 .

\subsection{Cross Section $\sigma_{\gamma \gamma}$}

The reliable description of the process $\gamma \gamma \rightarrow$ hadrons is very important not only from the theoretical point of view, but also since this process gives the bulk of background at future linear colliders. This cross section, $\sigma_{\gamma \gamma}$, can be related to the total cross sections for hadron production in collisions: pp, $\mathrm{p} \overline{\mathrm{p}}$, and $\gamma \mathrm{p}$ since all of them are dominated by the exchange of the Pomeron. Assuming the factorization and the Additive Quark Model the data for all these processes, after the appropriate rescaling, should lie on a universal curve. This is not observed - see Fig. 14-left, where a problem with the normalization of $\gamma \gamma$ data is indicated 41 . The high energy $\gamma \gamma$ data were checked to be consistent with each other, see Fig. 14-right, where all LEP data (together with PLUTO, TPC- $2 \gamma$ and MD-1 results) are presented as a function of $W_{\gamma \gamma}$ 43. The observed rising behaviour of cross sections can be obtained in e.g., minijet models. To ensure unitarity the Eikonal Minijet Model was applied

summary-ascona-final: submitted to World Scientific on March 9, 2022 17 

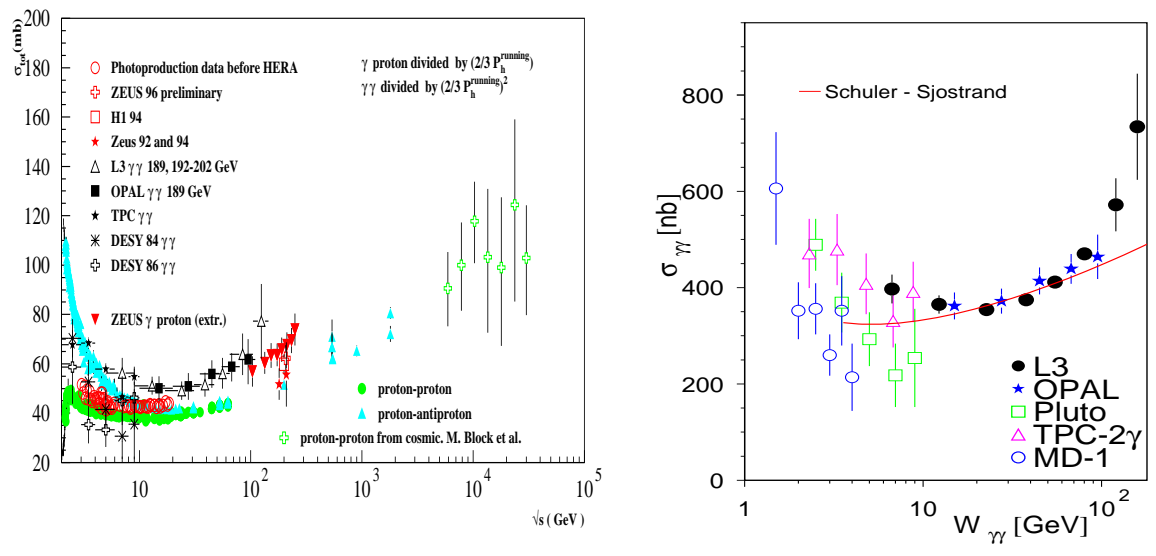

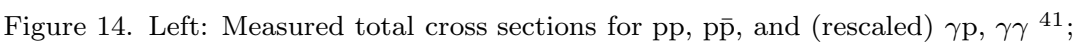
Right: LEP data for $\sigma_{\gamma \gamma} 4$.

to describe the data (Fig. 15-left) and it would appear that data for $\sigma(\gamma \gamma \rightarrow$ hadrons Lare overestimated by about $10 \%$. Effects of resummation were also studied 11 .

A DELPHI measurement of the $\sigma_{\gamma^{*} \gamma^{*}}$ for very low $Q^{2}$ double-tag eyents, important for a reliable estimation of the $\sigma_{\gamma \gamma}$, was also presented 4 , see Fig. 15-right for results.

\subsection{Cross Section $\sigma_{\gamma^{*} \gamma^{*}}$}

In the virtual photon collisions, $\gamma^{*} \gamma^{*} \rightarrow$ hadrons, with large virtualities of both photons, $Q_{1}^{2} \sim Q_{2}^{2}$, a large scale $Q_{1}^{2} \sim Q_{2}^{2} \sim Q^{2}$, allows for a clean test of perturbative QCD in Regge limit. Such measurements with the aim to test the BFKL picture, as DGLAP evolytion is suppressed for $Q_{1}^{2} \sim Q_{2}^{2}$, were performed by the LEP experiments 45,4 , see Fig. 16-left for results 45 . It is shown that LO BFKL can not describe the data, however, when the NLO corrections to BFKL expressions in the BLM optimal scale setting were implemented 40 a good agreement is found (Fig. 16-right).

summary-ascona-final: submitted to World Scientific on March 9, 2022 18 

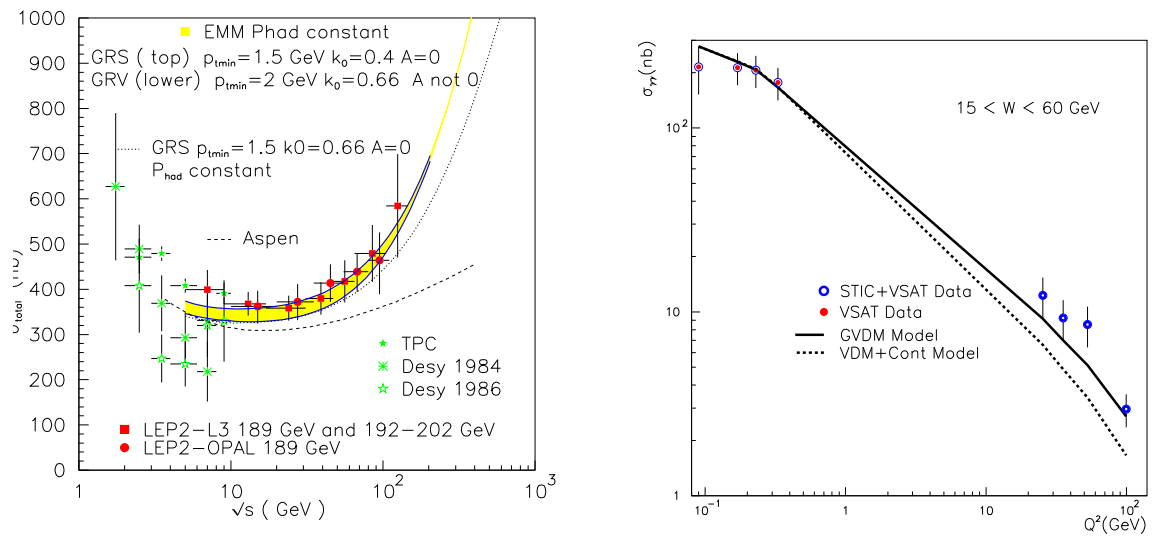

Figure 15. Left: Two-photon cross section $\sigma_{\gamma \gamma}$ versus $\sqrt{s}$ in prious models 4 ; Right: The DELPHI data for very low $Q^{2}$ double-tag events 44 .
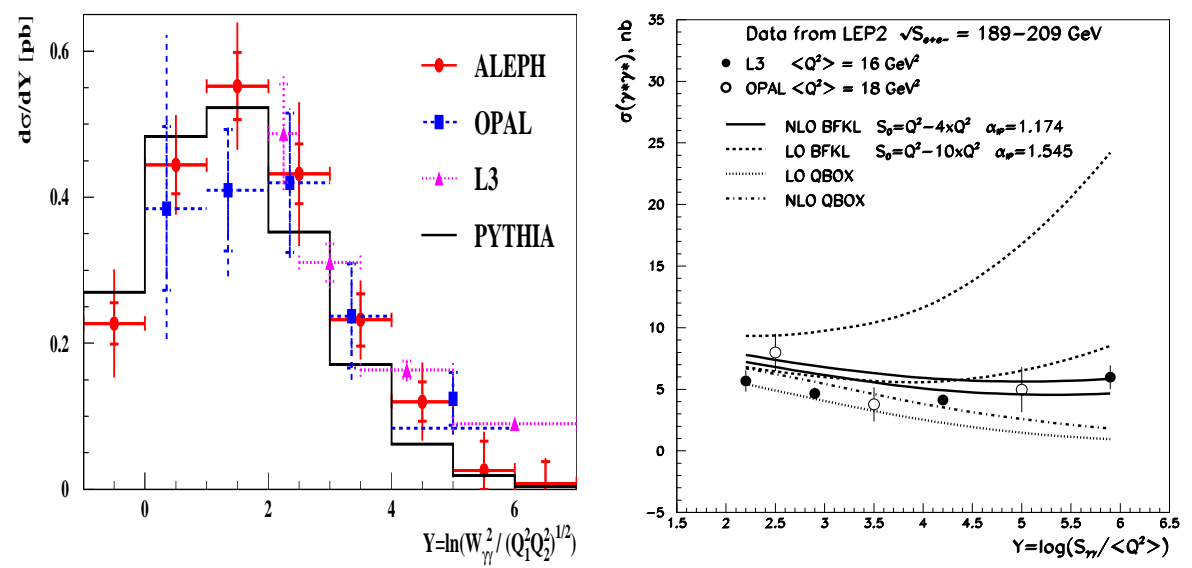

Figure 16. Cross section for $\gamma^{*} \gamma^{*} \rightarrow$ hadrons as function of $Y=\ln W_{\gamma \gamma}^{2} / Q^{2}$. Left: The LEP 2 data, by ALEPH, L3 and OPAL, in comparison to PYTHIA 45 (the OPAL and L3 data are extrapolated to the acceptance range of ALEPH),

Right: Comparison of the LO and NLO BFKL predictions to L3 and OPAL data 40. 


\subsection{Diffraction at Low and High Virtuality of the Photon at HERA. $D V C S$}

Various models of diffraction exist (e.g., pure soft Regge exchange models, the resolved Pomeron model 47 and dipole models 4 ) and they can be tested by comparison of distributions in specific processes. The diffractive production of vector mesons were measured in the photoproduction and DIS $\mathrm{ep}_{\mathrm{e}}$ events at HERA. The photoproduction of vector mesons are shown in Fig. 17-left, where a steeper rise with energy is observed for heavy mesons as compared to light mesons 49 . For electroproduction the vector meson production ratios as a function of $Q^{2}$ were used to test the flavour independence hypothesis: $\rho: \omega: \phi: \mathrm{J} / \psi=9: 1: 2: 8$, or (slightly modified ratios predicted in pQCD). For the light vector mesons the data scale with $\left(Q^{2}+m^{2}\right)$, while the ones for $\mathrm{J} / \psi$ do not. In Fig. 17-right the rate for the $\rho$ production as a function of $Q^{2}$ is presented in a form of the ratio $R=\sigma_{L} / \sigma_{T}$, and found to be in agreement with QCD 49.

The Deeply Virtual Compton Scattering (DVCS) process, $\gamma^{*} p \rightarrow \gamma p$, is an exclusive analogue to the prompt $\gamma$ production in $\mathrm{DIS}_{\mathrm{ep}}: \gamma^{*} \mathrm{p} \rightarrow \gamma \mathrm{X}$. In DVCS scales are different: $Q^{2}$ is much larger than $\left(p_{T}^{\gamma}\right)^{2}$, and $|t|$ is smaller than $1 \mathrm{GeV}^{2}$. This process can be described in terms of the skewed parton distributions, which can be treated as a generalization of the standard parton densities. The results of measurements at HERA (H1) for the DVCS cross section as a function of $Q^{2}$ and $W$ are presented in Fig. 18 .

In the HERMES experiment at HERA polarized $\mathrm{e}^{ \pm}$beams collide with the (gas) targets. The exclusive diffractive $\rho$ production in DIS $_{\text {ep }}$ events was analyzed. The results for $\sigma\left(\gamma_{L}^{*} \mathrm{p} \rightarrow \rho \mathrm{p}\right)$ are shown in Fig. 19 for two $Q^{2}$ samples $\left(\left\langle Q^{2}\right\rangle \approx 2\right.$ and $\left.4 \mathrm{GeV}^{2}\right)$. The quark exchange subprocess dominates for smaller $Q^{2}$ events, while gluon exchange mechanism starts to be important at larger $Q^{2}$. Spin effects in $\rho$ electroproduction were studied with the conclusion that s-channel helicity consprvation is slightly violated. The double-spin asymmetry were also studied 50 (for DIS and photoproduction) and results were interpreted in terms of GVMD.

\section{Exclusive Channels and Resonances in $\gamma \gamma$ collisions}

Study of pure leptonic final states in $\gamma \gamma$ collision were performed to test QED (up to $\mathcal{O}\left(\alpha^{4}\right)$ ) but also to demonstrate the understanding of the detectors. The remarkable number of new results on exclusive hadronic final states presented during the conference, some of them from new high luminosity low energy colliders, leads to better understanding of strong interaction at low

summary-ascona-final: submitted to World Scientific on March 9, 2022 20 


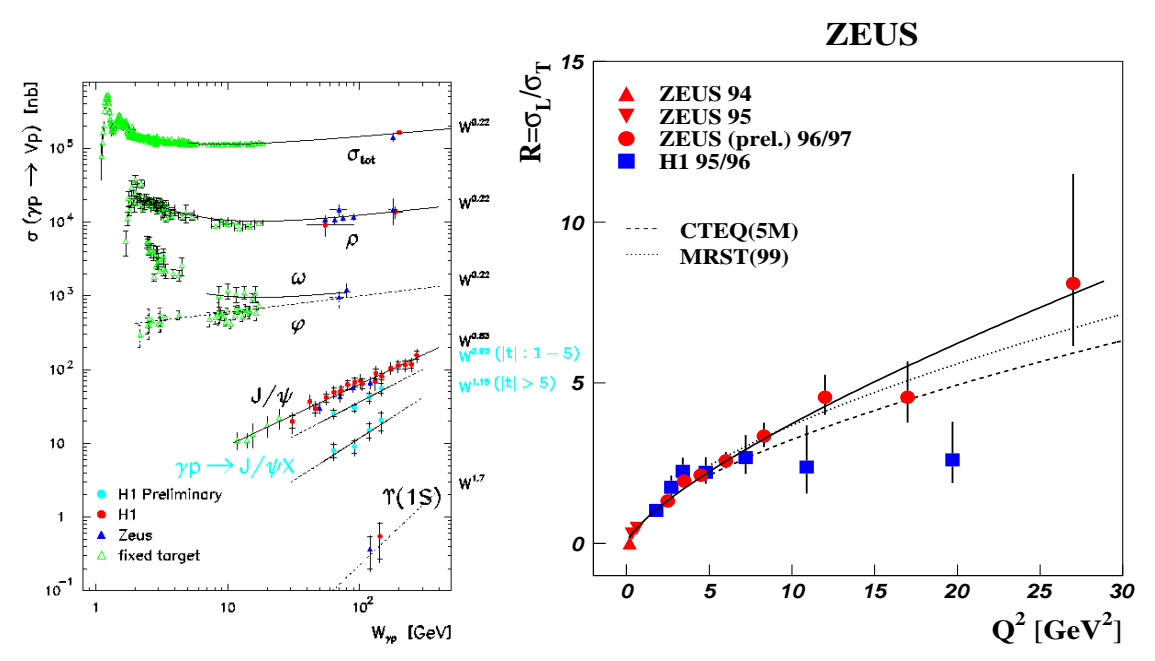

Figure 17. Left: Photoproduction of vector mesons at HERA and fixertarget exp. 40, Right: The ratio $R=\sigma_{L} / \sigma_{T}$ for the $\rho$ production as a function of $Q^{2} 49$.
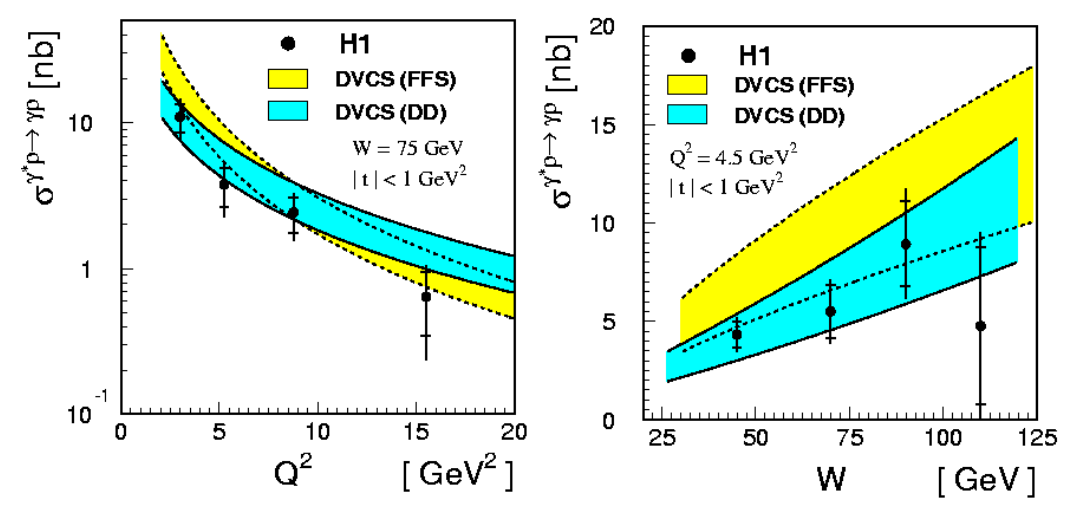

Figure 18. Cross sections for DVCS as function of $Q^{2}$ and $W$, from $\mathrm{H} 19$.

energies. If a large momentum scale is involved in the reaction, then we can use pQCD assuming factorization of the amplitude into a perturbative and non-perturbative part 51 . In Refs. 52 the meson distribution amplitude were constrained from study of transition $\gamma^{*} \gamma \rightarrow \pi, \eta, \eta^{\prime}$. Radiative decay ampli-

summary-ascona-final: submitted to World Scientific on March 9, 2022 21 


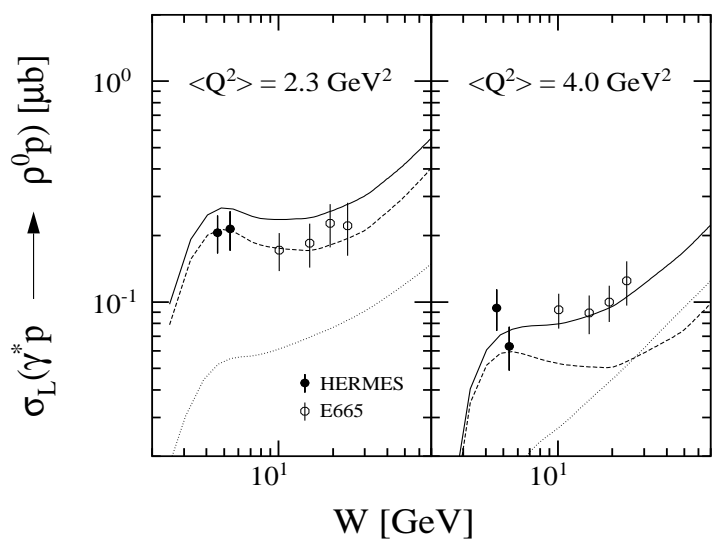

Figure 19. HERMES data for $\rho$ production: $\sigma\left(\gamma_{L}^{*} \mathrm{p} \rightarrow \rho \mathrm{p}\right)$ for two $\Omega^{2}$ samples- dotted (dashed) line corresponds to the gluon (quark) exchange subprocesses 50 .

tudes for various particles, among them the decays $\pi, \eta, \eta^{\prime} \rightarrow \gamma^{*} \gamma$, lead to reconstruction of pion and photon wave functions 53 .

Production of particles containing heavy quarks is of special interest; results obtained at $\mathrm{e}^{+} \mathrm{e}^{-}$colliders (see below) and at HERA for the production of excited $\mathrm{P}$-wave charm mesons 54 were discussed during the conference.

One of the basic open question of QCD is the existence of the glueballs, a bound state of gluons. The two-photon process is a powerful anti-filter for glueballs. The lattice simulation 55 of the quenched glueball spectrum was preformed with first promising results for $n_{f}=2$.

\subsection{Lepton and Hadron Pairs}

Lepton pair production $\mathrm{e}^{+} \mathrm{e}^{-} \rightarrow \mathrm{e}^{+} \mathrm{e}^{-} \ell^{+} \ell^{-}(\ell=\mu, \tau)$ have been measured by L3 at LEP 2 as function of centre-of-mass energy and of $W_{\gamma \gamma}$. Limits on a possible anomalous coupling of the $\tau$ have been given 56 .

New LEP 2 results on pion and kaon pair production were presented 57 and compared with the QCD-inspired calculation of Brodski and Lepage (Fig. 20). ALEPH and DELPHI find good agreement for kaon pairs both in normalization and shape of the distribution in scattering angle $\cos \theta^{*}$ and $W_{\gamma \gamma}$. For the pions the shape of the distributions all agree, while for the normalization, sensitive to nonperturbative input, neither the experiments nor experiments versus theory agree.

The production mechanism for dibaryon production was tested by OPAL

summary-ascona-final: submitted to World Scientific on March 9, 2022 22 

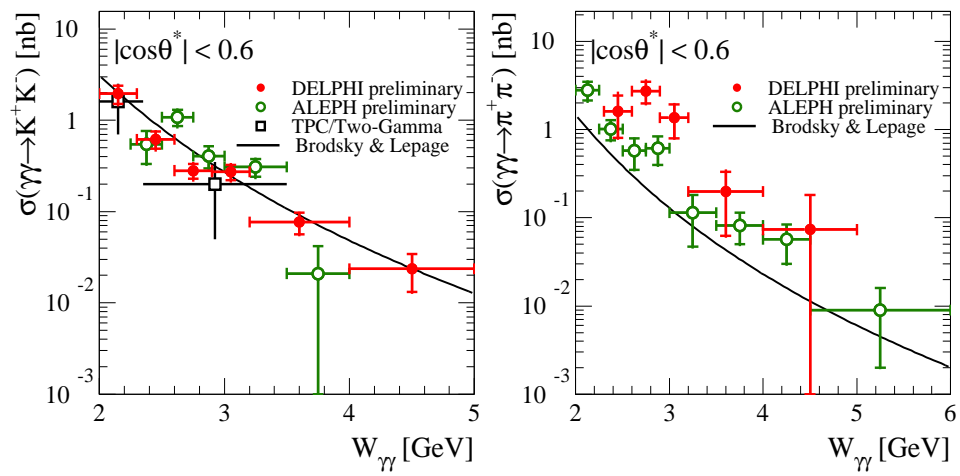

Figure 20. Differential cross section for $\gamma \gamma$ to two charged ond pion pairs, respectively as function of $W_{\gamma \gamma}$ as measured by DELPHI and ALEPH 5 .

and Belle in p p and by L3 in $\Lambda \bar{\Lambda}$ final states. The dibaryon mass spectra as function of $W_{\gamma \gamma}$ (from the dimensional counting rule $\sim s^{-n}$ is expected) prefer the quark-diquark model $(n=4)$ rather than the three-quark model $(n=6)$. The measurements are in agreement with previous measurements, though all predictions tend to lie below the data 58

\subsection{Exclusive Meson Production and Glueball Search}

The results are manifold and involved. The branching ratios, production as function of the virtuality of the photon and form factors of specific mesons are interesting on their own, but also constrain the gluonium content of the particle. The determination of the spin and helicity of resonances has been performed, showing that for the tensor mesons the multiplet is understood, while for the scalars the situation is more unclear. Possible mixture of states complicates the interpretation and the isolation of the best glueball candidate 59.00 .

\subsection{Charmonia and Bottomonia}

Extraction of $\Gamma_{\gamma \gamma}\left(\eta_{\mathrm{c}}\right)$ was investigated in the $\pi^{+} \pi^{-} \mathrm{K}^{+} \mathrm{K}^{-}, 2\left(\mathrm{~K}^{+} \mathrm{K}^{-}\right)$, $\mathrm{K}_{\mathrm{S}} \mathrm{K}^{+} \pi^{-}$and $2\left(\pi^{+} \pi^{-}\right)$decay modes by DELPHI. While the combination of the first three channel results in a two-photon width of $13.0 \pm 2.7 \pm 5.0 \mathrm{keV}$ and is compatible, though somewhat higher, with previous measurements, the non-observation of the $4 \pi$ decay leads to an upper limit of $3.8 \mathrm{keV} 60$.

summary-ascona-final: submitted to World Scientific on March 9, 2022 23 


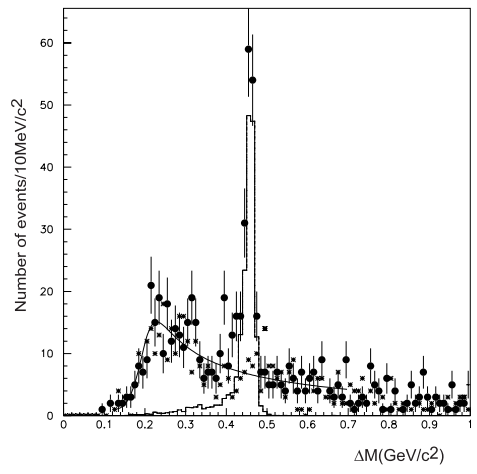

Figure 21. Mass difference $m\left(\ell^{+} \ell^{-} \gamma\right)-m\left(\ell^{+} \ell^{-}\right)$for $\chi_{\mathrm{c} 0} \rightarrow \mathrm{J} / \psi$ events as measured by Belle 62

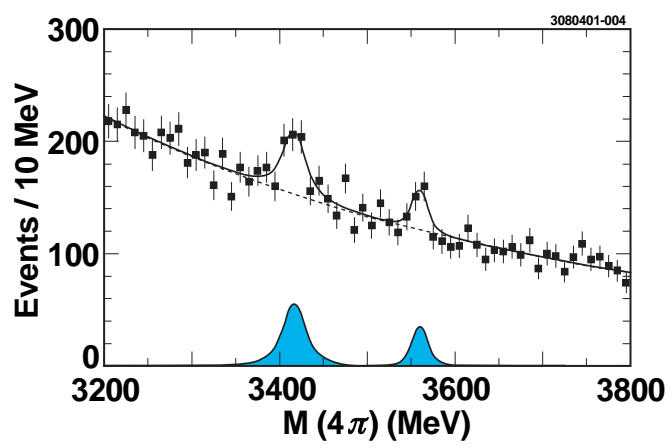

Figure 22. Invariant mass distributipn of four charged pions as measured by CLEO 61 .

The CLEO Collaboration presents a first observation of the $\chi_{\mathrm{c} 0}$ in twophoton collisions 61 . A result of $\Gamma_{\gamma \gamma}\left(\chi_{\mathrm{c} 0}\right)=3.76 \pm 0.65 \pm 1.81 \mathrm{keV}$ is extracted in the $\pi^{+} \pi^{-} \pi^{+} \pi^{-}$decay mode. Belle shows that the $\chi_{\mathrm{c} 0}$ is present in the $\mathrm{K}_{\mathrm{S}} \mathrm{K}_{\mathrm{S}}$ mass spectrum as well 62 .

Belle also measures the $\chi_{\mathrm{c} 2}$ production in the $\mathrm{J} / \psi \gamma$ decay mode (Fig. 21), CLEO presents a first measurement from the $\pi^{+} \pi^{-} \pi^{+} \pi^{-}$decay mode (Fig. 22). Both results are lower, but in agreement with previous results in two-photon collisions and larger than the results from $\mathrm{p} \overline{\mathrm{p}}$ collisions. As pointed out 61 the ratio $\Gamma_{\gamma \gamma}\left(\chi_{\mathrm{c} 0}\right) / \Gamma_{\gamma \gamma}\left(\chi_{\mathrm{c} 2}\right)$ is a good QCD test with small theoretical uncertainties and could provide a measurement of $\alpha_{\mathrm{S}}$, but the calculations of the $\mathcal{O}\left(\alpha_{\mathrm{S}}^{2}\right)$ term for the $\chi_{\mathrm{c}}$ states are badly needed.

A first search for the $\eta_{\mathrm{b}}$ meson in two-photon collisions was performed in ALEPH 63, the bottomonium ground state being still undetected. The observation of one $\eta_{\mathrm{b}}$ candidate $\left(m=9.30 \pm 0.02 \pm 0.02 \mathrm{GeV} / c^{2}\right)$ compatible with background gives upper limits of $57 \mathrm{eV}$ and $128 \mathrm{eV}$ for $\Gamma_{\gamma \gamma}\left(\eta_{\mathrm{b}}\right) \times \mathrm{BR}$ for decays to 4 and 6 charged particles, respectively.

\section{Future Projects and Related Topics}

The two-photon collisions at the future LHC collider may turn out to be important for the search of the Higgs boson, as addressed in the presentation 65 . The coherent photon-pomeron and photon-photon interactions appear already in ultraperipheral collisions at BHIC; a clear $\rho^{0}$ signal was observed in the $\pi^{+} \pi^{-}$invariant mass spectrum 66 .

summary-ascona-final: submitted to World Scientific on March 9, 2022 24 
Relativistic nuclear collisions at LHC and RHIC were studied in 67. In particular the Coulomb and unitarity corrections to the single $\mathrm{e}^{+} \mathrm{e}^{-}$pair production as well as the cross section $\sigma_{n}$ for the multiple pair production were obtained in an analytic form. Some of the results differ from results published by other authors. The Born cross section for $\mathrm{e}^{+} \mathrm{e}^{-}$pair production is given by:

$$
\sigma_{\text {Born }}=\frac{28}{27 \pi} \frac{\left(Z_{1} Z_{2} \alpha^{2}\right)^{2}}{m_{\mathrm{e}^{2}}}\left[L^{3}-2.198 L^{2}+3.821 L-1.632\right],
$$

with $L=\ln \left(\gamma_{1} \gamma_{2}\right)$, where $\gamma_{i}$ and $Z_{i}$ are the Lorentz factors and the charges of the colliding nuclei. The cross sections for this process at LHC and RHIC are huge, so the pair production can be a serious background. The control of this background may turn out important for a good beam lifetime and luminosity of the colliders. In Table 1 results for $\sigma_{\text {Born }}$ are given together with corrections (calculated for small $1 / L$ in the main logarithmic approximation).

Table 1. Results for relativistic heavy-ion colliders $\left(Z_{1}=Z_{2} \equiv Z\right.$ and $\left.\gamma_{1}=\gamma_{2} \equiv \gamma\right)$

\begin{tabular}{|c|c|c|c|c|c|}
\hline Collider & $Z$ & $\gamma$ & $\sigma_{\text {Born }}[\mathrm{kb}]$ & $\frac{\sigma_{\text {Coul }}}{\sigma_{\text {Born }}}$ & $\frac{\sigma_{\text {unit }}}{\sigma_{\text {Born }}}$ \\
\hline RHIC, $\mathrm{Au}-\mathrm{Au}$ & 79 & 108 & 36.0 & $-25 \%$ & $-4.1 \%$ \\
\hline LHC, $\mathrm{Pb}-\mathrm{Pb}$ & 82 & 3000 & 227 & $-14 \%$ & $-3.3 \%$ \\
\hline LHC, $\mathrm{Ca}-\mathrm{Ca}$ & 20 & 3700 & 0.872 & $-1.06 \%$ & $-0.025 \%$ \\
\hline
\end{tabular}

Very important for the investigations and the understanding of the photon and its interactions are planned $\mathrm{e}^{+} \mathrm{e}^{-}$Linear Colliders (LC) at large CM energy (500, $800 \mathrm{GeV}$ or higher), in particular the Photon Collider (PC) option based on Compton backscattering of laser light off the high energy electrons, Such $\gamma \gamma$ and $e \gamma$ collider has unique properties as it was discussed in Refs. 68.69 (with particular emphasis on the TESLA PC 00:71). Cross sections are higher than for corresponding processes in $\mathrm{e}^{+} \mathrm{e}^{-}$collision, accessible masses are larger and there are unique reactions, such as $\gamma \gamma \rightarrow \mathrm{H}$. Since the $\gamma \gamma$ luminosity in the high energy part of spectra at TESLA can be about $30 \%$ of the $\mathrm{e}^{+} \mathrm{e}^{-}$ luminosity (see also Ref. 72 for a fast luminosity measurements study) the number of "interesting" events at the photon collider will be even higher than in $\mathrm{e}^{+} \mathrm{e}^{-}$one. A short list of physics processes for the photon collider is presented in Table 201 .

summary-ascona-final: submitted to World Scientific on March 9, 2022 25 
Table 2. Gold-plated processes at photon colliders

\begin{tabular}{|c|c|}
\hline Reaction & Rer \\
\hline $\begin{array}{l}\gamma \gamma \rightarrow h_{0} \rightarrow \overline{\mathrm{b}} \mathrm{b}, \gamma \gamma \\
\gamma \gamma \rightarrow h_{0} \rightarrow W W\left(W W^{*}\right) \\
\gamma \gamma \rightarrow h_{0} \rightarrow \mathrm{ZZ}\left(\mathrm{ZZ}^{*}\right)\end{array}$ & $\begin{array}{c}M_{h_{0}}<160 \mathrm{GeV} \\
140<M_{h_{0}}<190 \mathrm{GeV} \\
180<M_{h_{0}}<350 \mathrm{GeV}\end{array}$ \\
\hline $\begin{array}{l}\gamma \gamma \rightarrow H, A \rightarrow \overline{\mathrm{b}} \mathrm{b} \\
\gamma \gamma \rightarrow \tilde{f} \tilde{\tilde{f}}, \tilde{\chi}_{i}^{+} \tilde{\chi}_{i}^{-}, H^{+} H^{-} \\
\gamma \gamma \rightarrow S[\tilde{\tilde{t}}] \\
\gamma \mathrm{e} \rightarrow \tilde{e}^{-} \tilde{\chi}_{1}^{0}\end{array}$ & $\begin{array}{c}\mathcal{M S S M} \text { heavy Higgs } \\
\text { supersymmetric particles } \\
\tilde{\tilde{t} \tilde{t}} \text { stoponium } \\
M_{\tilde{e}^{-}}<0.9 \times 2 E_{0}-M_{\tilde{\chi}_{1}^{0}}\end{array}$ \\
\hline $\begin{array}{l}\gamma \gamma \rightarrow W^{+} W^{-} \\
\gamma \mathrm{e}^{-} \rightarrow W^{-} \nu_{e} \\
\gamma \gamma \rightarrow W W+W W(Z Z)\end{array}$ & $\begin{array}{c}\text { anom. } W \text { inter., extra dim. } \\
\text { anom. } W \text { couplings } \\
\text { strong } W W \text { scattering }\end{array}$ \\
\hline $\begin{array}{l}\gamma \gamma \rightarrow t \bar{t} \\
\gamma \mathrm{e}^{-} \rightarrow \bar{t} b \nu_{e}\end{array}$ & $\begin{array}{c}\text { anom. } t \text {-quark interactions } \\
\text { anom. } W t b \text { coupling }\end{array}$ \\
\hline $\begin{array}{l}\gamma \gamma \rightarrow \text { hadrons } \\
\gamma \mathrm{e}^{-} \rightarrow e^{-} X \text { and } \nu_{e} X \\
\gamma g \rightarrow \overline{\mathrm{q}} \mathrm{q}, \overline{\mathrm{cc}} \\
\gamma \gamma \rightarrow J / \psi J / \psi\end{array}$ & $\begin{array}{c}\text { total } \gamma \gamma \text { cross section } \\
\text { struct. functions } \\
\text { gluon distr. in the photon } \\
\text { QCD Pomeron }\end{array}$ \\
\hline
\end{tabular}

The unique feature of a $\gamma$ e collider is the possibility of the measurement of the structure function for the real photon (not quasi-real as presently), in particular the spin-dependent structure functions, not measured so far.

The future of exclusive particle production measurements at charm and bottom factories (CLEO-c, BaBar and Belle experiments) were also discussed at the conference. E.g., at CLEO-c the high statistics and high precision measurements allow progress in measuring branching ratios, form factors, decay constants, CKM matrix parameters etc. 64 .

\section{Conclusion}

Plenty of new high quality data involving photons in hadronic processes have been analysed after PHOTON 2000 at LEP and HERA, as well as from experiments at Tevatron, RHIC, VEPP-2M, DAPHNE, CESR, KEKB and SLAC. An impressive progress in the measurements is accompanied by improvements in the theoretical descriptions of hard, semi-hard and soft hadronic processes involving photons. Also pure QED processes were studied, relevant for present and future colliders.

Processes with photons provide simple tests of new theoretical ideas, like

summary-ascona-final: submitted to World Scientific on March 9, 2022 26 
e.g., non-commutative QED; in future conferences of the PHOTON series, we certainly will learn more about these new possible interactions of the photon so that we finally understand "what are light quanta".

\section{Acknowledgements}

It is pleasure for us to thank the organizers of the conference PHOTON2001, especially Maria Kienzle and Maneesh Wadhwa, for a nice atmosphere and perfect organization in the beautiful site of Ascona.

Armin Böhrer thanks the Bundesministerium für Bildung und Forschung of Germany (grant BMBF 05 HE 1 PS A8) for the financial support. Maria Krawczyk is grateful to the organizers of the conference and the Polish Com-

mittee for Scientific Research (grant 2P03B05119 (2002)) for the financial support.

\section{References}

1. Proceedings of the International Conference on the Structure of the Photon (PHOTON 2000), Ambleside (2000), Eds.: A. Finch, New York, American Institute of Physics.

2. A. de Roeck, Survey in High Energy Physics, Vol.16, 49 (2001).

3. R. Nisius, Phys. Rep. 332, 165 (2000); M. Krawczyk et al., Phys. Rev. 345, 265 (2001); M. Whalley, J. Phys. G27, 12 A1 (2001); M. Erdmann, Springer Tracks in Modern Physics 138 (1997), Heidelberg, Springer.

4. R. Nisius, these proceedings and private communication.

5. R. Taylor, these proceedings.

6. I. Tyapkin, these proceedings.

7. P. Jankowski, these proceedings.

8. A. Csilling, these proceedings.

9. I. Fleck, these proceedings.

10. M. Wing, these proceedings.

11. M. Vasquez, these proceedings.

12. B. Pötter, these proceedings.

13. T. Schörner, these proceedings.

14. A. Böhrer, Phys. Rep. 291, 107 (1997); I.G. Knowles and G.D. Lafferty, J. Phys. G23 731 (1997).

15. D. Traynor; S. Boogert, these proceedings.

16. S. Padhi, these proceedings.

17. T. Wengler, these proceedings.

18. J. Masik, these proceedings.

summary-ascona-final: submitted to World Scientific on March 9, 2022 27 
19. U. Jezuita-Dabrowska, these proceedings.

20. A. Valkarova, these proceedings.

21. K. Sedlak, these proceedings.

22. M. Begel, these proceedings.

23. J. Lillich, these proceedings.

24. S. Tapprogge, these proceedings.

25. J. Breitweg et al. [ZEUS Collaboration], Phys. Lett. B 472, 175 (2000).

26. M. Fontannaz et al., Eur. Phys. J. C 21, 303 (2001); A. Zembrzuski and M. Krawczyk, Phys. Rev. D 64, 114017 (2001).

27. S. Chekanov et al. [ZEUS Collaboration], Phys. Lett. B 511, 19 (2001).

28. P. Achard, these proceedings.

29. H. Jung, Phys. Rev. D 65, 034015 (2002); DESY-01-136, hepph/0110034 and these proceedings.

30. J. Chyla, these proceedings.

31. S. Frixione, these proceedings.

32. V. Andreev, these proceedings and private communication.

33. A. Sokolov, these proceedings.

34. B. West, these proceedings.

35. W. Erdmann, these proceedings.

36. M. Turcato, these proceedings.

37. M. Klasen et al., Nucl. Phys. B 609, 518 (2001).

38. S. Passaggio, these proceedings.

39. V. Fadin, these proceedings.

40. V. Kim, these proceedings, S. Brodsky et al., hep-ph/9901229v3, V. Kim et al., hep-ph/9911241.

41. G. Pancheri, these proceedings.

42. T. Berndt, these proceedings.

43. M. Kienzle, these proceedings.

44. A. Nygren, these proceedings.

45. G. Prange,these proceedings.

46. C. H. Lin, M. Przybycień, these proceedings.

47. G. Ingelman and P. Schlein, Phys. Lett. B 152, 256 (1985).

48. K. Golec-Biernat and M. Wüsthoff, Phys. Rev. D 59, 014017 (1999).

49. S. Kananov, these proceedings.

50. K. Lipka, these proceedings.

51. G. P. Lepage and S. J. Brodsky, Phys. Rev. D 22, 2157 (1980).

52. P. Kroll, these proceedings.

53. A. V. Anisovich, these proceedings.

54. U. Karshon, these proceedings.

55. G. Bali, these proceedings.

summary-ascona-final: submitted to World Scientific on March 9, 2022 28 
56. G. Debreczeni; D. Haas, these proceedings.

57. K. Grzelak, these proceedings.

58. T. Barillari; A. Chen; B. Echenard, these proceedings.

59. C. Amsler, these proceedings.

60. S. Braccini, these proceedings.

61. H. Paar, these proceedings.

62. S. Uehara, these proceedings.

63. A. Böhrer, these proceedings.

64. D. Cassel, these proceedings.

65. K. Piotrzkowski, these proceedings.

66. F. Meissner, these proceedings.

67 . V.G. Serbo, these proceedings.

68. V. Telnov, these proceedings.

69. I. Ginzburg, D. Anipko, these proceedings.

70. R. Brinkmann et al. (Eds.), TESLA Technical Design Report, DESY2001-011 (2001)

71. B. Badelek et al. (Eds.) [ECFA/DESY Photon Collider Working Group], part VI, appendices, chapter 1 . The Photon Collider at TESLA, hepex/0108012;

Proceedings of Photon Collider Workshop, Hamburg (2000), Eds.: R.D. Heuer et al., Nucl. Instrum. Methods A 472, 1 (2001);

72. W. da Silva, these proceedings

summary-ascona-final: submitted to World Scientific on March 9, 2022 29 